\title{
Efficient 3D Endfiring TRUS Prostate Segmentation with Globally Optimized Rotational Symmetry
}

\author{
Jing Yuan, Wu Qiu, Eranga Ukwatta, Martin Rajchl \\ Robarts Research Institute \\ Western University, London, ON Canada \\ \{jyuan, wqiu, eukwatta, mrajchl\}erobarts.ca
}

\author{
Xue-Cheng Tai \\ Mathematics Department \\ University of Bergen, Bergen, Norway \\ tai@math.uib.no
}

\author{
Aaron Fenster \\ Robarts Research Institute \\ Western University, London, ON Canada \\ afenster@robarts.ca
}

\begin{abstract}
Segmenting 3D endfiring transrectal ultrasound (TRUS) prostate images efficiently and accurately is of utmost importance for the planning and guiding 3D TRUS guided prostate biopsy. Poor image quality and imaging artifacts of 3D TRUS images often introduce a challenging task in computation to directly extract the $3 D$ prostate surface. In this work, we propose a novel global optimization approach to delineate $3 D$ prostate boundaries using its rotational resliced images around a specified axis, which properly enforces the inherent rotational symmetry of prostate shapes to jointly adjust a series of $2 D$ slicewise segmentations in the global 3D sense. We show that the introduced challenging combinatorial optimization problem can be solved globally and exactly by means of convex relaxation. In this regard, we propose a novel coupled continuous max-flow model, which not only provides a powerful mathematical tool to analyze the proposed optimization problem but also amounts to a new and efficient duality-based algorithm. Extensive experiments demonstrate that the proposed method significantly outperforms the state-of-art methods in terms of efficiency, accuracy, reliability and less user-interactions, and reduces the execution time by a factor of 100 .
\end{abstract}

\section{Introduction}

Prostate adenocarcinoma (PCa) is one of the most frequently diagonosed cancers in North America with over 200,000 new cases diagnosed each year [11]. Currently, $3 \mathrm{D}$ endfiring transrectal ultrasound (TRUS) is the most commonly used imaging modality for image-guided biopsy of PCa due to its real-time imaging capability, low cost and simplicity [15]. Segmenting the acquired 3D endfiring TRUS prostate images efficiently and accurately, especially in an automated or semi-automated way, is highly desired in a 3D endfiring TRUS guided prostate biopsy system [3], due to the arduous and time consuming effort associated with manual 3D prostate segmentation. In addition, through the segmentation results of 3D prostate TRUS and MR images, the 3D surface-based TRUS-MRI registration technique provides an effective way to target biopsy needles toward regions of the prostate containing MR identified suspicious lesions, as an alternative to the more expensive and inefficient, MRI-based prostate biopsy [5, 12]. However, poor TRUS image quality, such as US speckle, shadowing by calcifications, missing edges or texture similarities between the inner and outer regions of the prostate [22] etc, makes it challenging to implement an automated or semiautomated $3 \mathrm{D}$ prostate TRUS segmentation in practice.

\subsection{Previous Approaches}

In general, the proposed automated or semi-automated approaches to 3D TRUS prostate segmentation can be summarized by two categories: the direct 3D segmentation methods and 3D resliced segmentation methods. Essentially, most direct 3D segmentation methods [10, 17, 13, 9] propose to evolve an initialized 3D surface to the correct prostate boundaries, with or without optimized shape deformations, which worked well for the reported applications. However, direct computation over the large 3D TRUS data volumes makes them significantly time-consuming; moreover, intensive user interactions are required to initialize and conduct such direct 3D segmentation approaches. In contrast, 3D resliced segmentation methods "cut" the input 3D TRUS image into a parallel or rotational sequence of 
$2 \mathrm{D}$ slices and reduce the $3 \mathrm{D}$ US segmentation to a series of 2D slicewise segmentations, while the spatial correlation between two adjacent slicewise segmentations is enforced. Comparing to direct 3D segmentation methods, such 3D resliced methods enjoy some significant advantages in numerics: clearly, each of the reduced 2D segmentation subproblems is much simpler than the original 3D segmentation problem, and, it is also much computationally cheaper to incorporate $2 \mathrm{D}$ shape information into the $3 \mathrm{D}$ segmentation procedure. Especially, the rotational-resliced segmentation approaches, recently proposed in [8, 14], sever the input 3D TRUS image into $n$ slices with equal angular spacing about the specified rotational scan axis, as illustrated in Fig.1(a), and make use of the approximate rotational symmetry of prostate shapes around the given axis to assist the 3D prostate segmentation. Since most information about the prostate boundary is available in every single rotationalresliced 2D image, these methods successfully avoid the difficulties of extracting the correct prostate base and apex, which is encountered by the other approaches; thus, improved segmentation accuracy and robustness.

The rotational-resliced segmentation approaches [8, 14] propose to first segment the initial slice and propagate its result as a spatial region constraint to facilitate segmenting its adjacent slice in clockwise or counter-clockwise direction; then, repeat propagating the obtained result as the region prior to the segmentation of the next adjacent slice and so on, until all the slices are segmented. The 3D prostate surface is, therefore, reconstructed from all the extracted 2D contours. However, such 'propagation' procedures often carry the following drawbacks: first, the segmentation errors appearing in one slice are also propagated to the segmentation of its following slices, thus causing an accumulated error in all the succeeded segmentations; second, the segmentation result of any slice has no effect on refining the segmentation of its preceded slices, hence it is impossible to adjust all the 2D slicewise segmentations jointly in a global way to improve the segmentation accuracy and robustness; last but not least, sequentially segmenting the $n$ 2D slices is not efficient in numerics, and each slicewise segmentation is implemented by active contour [8] or level-set [14], whose results highly depend on the initializations and are often trapped by a local optimum.

\subsection{Contributions}

In this paper, we propose a novel global optimization approach to segmenting 3D rotational-resliced prostate TRUS images, which successfully addresses the main disadvantages of the previous approaches by simultaneously segmenting the $n$ 2D image slices while globally optimizing the introduced rotational symmetry prior. We show that the introduced challenging combinatorial optimization problem can be globally optimized by means of convex relaxation. In addition, we introduce the novel coupled continuous maxflow model as the dual formulation of the given convex relaxed optimization problem. With help of the new coupled continuous max-flow model, we prove the proposed combinatorial optimization problem can be solved globally and exactly. Hence, the globally optimal segmentation of the $n$ $2 \mathrm{D}$ slices w.r.t. the rotational symmetry prior can be accordingly achieved. Meanwhile, we derive a new and efficient coupled continuous max-flow based algorithm by the modern convex optimization theory, which can be directly implemented on GPU for a substantial speed-up in computation. Extensive experiments demonstrate that the proposed method significantly outperforms the state-of-art methods in terms of efficiency, accuracy and reliability, with less interactions and reducing execution time by a factor of 100 . Moreover, the learned 2D shape prior can be easily integrated into the proposed method.

The conception of continuous max-flow, proposed and used in this study and [20, 21, 2], is name-wise similar to continuous maximal flow introduced in [1]; but it stems from a different optimization theory in terms of convex duality, hence derives the different algorithm scheme based on augmented multipliers.

\section{Global Optimization to 3D Prostate TRUS Segmentation}

Let $\mathcal{V}$ be the input 3D prostate endfiring transrectal ultrasound (TRUS) image, which is resliced rotationally around a given axis to $n 2 \mathrm{D}$ images $\mathcal{S}_{1} \ldots \mathcal{S}_{n}$. The ellipsoid-like shape of the prostate allows to specify the resliced rotation axis, such that the extracted prostate regions on every two adjacent slices are spatially consistent, namely the rotational symmetry prior. In this section, we propose a novel and efficient global optimization approach to simultaneously extract the $n$ prostate contours of the input slices $\mathcal{S}_{1}$ $\ldots \mathcal{S}_{n}$ by jointly enforcing their rotational symmetry prior. We show the proposed combinatorial optimization problem can be exactly and globally solved by convex relaxation; for which we introduce a new spatially continuous max-flow model and prove its equivalence to the convex relaxed optimization formulation under the perspective of primal and dual. We demonstrate that the new spatially continuous max-flow model carries great advantages to the proposed 3D prostate TRUS segmentation approach in both optimization analysis and algorithmic scheme.

\subsection{Optimization Formulation with Rotational Symmetry Prior}

Slicewise Continuous Min-Cut Formulation Let $\mathcal{R}_{i}, i=$ $1 \ldots n$, denote the prostate region of the $2 \mathrm{D}$ slice $\mathcal{S}_{i}$, and $u_{i}(x), i=1 \ldots n$, be the labeling function of the prostate 


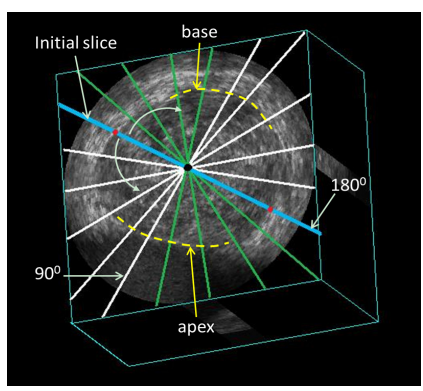

(a)

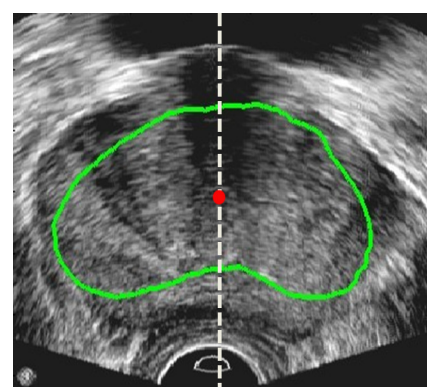

(b)

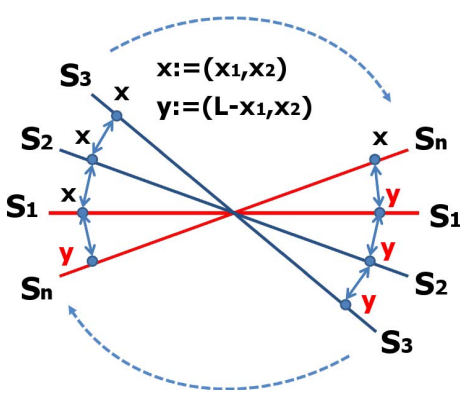

(c)

Figure 1. Initialization: (a) reslice the 3D prostate image; (b) rotation-axis (white dash-line) is specified to reslice the input 3D image and align each 2D slice; (c) the illustration of $\mathrm{n}$ slices from the top view: the initial slice and the last slice are colored in red and spatially correlated by left-right flipping, where $L$ is the length from the left side to the right side.

region $\mathcal{R}_{i}$ such that

$$
u_{i}(x)=\left\{\begin{array}{ll}
1, & \text { for } \forall x \in \mathcal{R}_{i} \\
0, & \text { otherwise }
\end{array}, \quad i=1 \ldots n .\right.
$$

The segmentation of each slice $\mathcal{S}_{i}, i=1 \ldots n$, can be formulated as the spatially continuous min-cut problem which minimizes the following energy function

$E_{i}\left(u_{i}\right):=\left\langle 1-u_{i}, C_{i}^{s}\right\rangle+\left\langle u_{i}, C_{i}^{t}\right\rangle+\int_{\Omega} g_{i}(x)\left|\nabla u_{i}\right| d x$

over the corresponding labeling function $u_{i}(x) \in\{0,1\}$. In (1), for each slice $\mathcal{S}_{i}, i=1 \ldots n$, two cost functions $C_{i}^{s}(x)$ and $C_{i}^{t}(x)$ are defined, which evaluate the costs to label the pixel $x \in \mathcal{S}_{i}$ as the prostate region and background respectively; the weighted total-variation function measures the length of each region indicated $u_{i}(x) \in\{0,1\}$.

Rotational Symmetry Prior In this work, the $n$ slices $\mathcal{S}_{1}$ $\ldots \mathcal{S}_{n}$ are simply aligned along the resliced rotation axis, such that the rotation axis vertically bisects each slice (see the white dot line in Fig.1(b)). We propose to enforce the rotational symmetry prior of the segmented prostate regions $\mathcal{R}_{i}, i=1 \ldots n$, by penalizing the spatial inconsistence of the extracted regions within two neightbour slices (see Fig. 1(c)), i.e.

$$
\left(\mathcal{S}_{1}, \mathcal{S}_{2}\right),\left(\mathcal{S}_{2}, \mathcal{S}_{3}\right), \ldots,\left(\mathcal{S}_{n-1}, \mathcal{S}_{n}\right),\left(\mathcal{S}_{n}, \mathcal{S}_{1}\right) .
$$

Specifically, we penalize the area difference of two adjacent prostate regions, i.e. minimize

$$
\pi_{i}(u):=\int_{\Omega}\left|u_{i+1}-u_{i}\right| d x, \quad i=1 \ldots n-1,
$$

and the area difference of $\mathcal{R}_{n}$ and $\mathcal{R}_{1}$ within the last and first slices defined as

$$
\pi_{n}(u):=\int_{\Omega}\left|u_{n}\left(L-x_{1}, x_{2}\right)-u_{1}\left(x_{1}, x_{2}\right)\right| d x
$$

where $x:=\left(x_{1}, x_{2}\right)$ and the spatial comparison is performed by left-right flipping the horizontal coordinate of the last slice, as illustrated by the two red lines in Fig.1(c).

Optimization Formulation Now we propose to extract the 3D prostate surface from the input 3D image by segmenting the $n$ 2D image slices while incorporating their rotational symmetry prior. In view of (1), (2) and (3), it can be formulated as follows

$$
\min _{u_{1 \ldots n}(x) \in\{0,1\}} \sum_{i=1}^{n} E_{i}\left(u_{i}\right)+\alpha \sum_{i=1}^{n} \pi_{i}(u) .
$$

\subsection{Convex Relaxation and Coupled Continuous Max-Flow Model}

In this study, we show that the proposed optimization problem (4) can be globally and exactly solved by its convex relaxation

$$
\min _{u_{1 \ldots n}(x) \in[0,1]} \sum_{i=1}^{n} E_{i}\left(u_{i}\right)+\alpha \sum_{i=1}^{n} \pi_{i}(u)
$$

where the binary-valued constraints $u_{1 \ldots n}(x) \in\{0,1\}$ in (4) is replaced by its convex relaxation $u_{1 \ldots n}(x) \in[0,1]$. Hence, (5) amounts to a convex optimization problem for which a global optimum exists.

We now study the convex relaxation problem (5) under the primal and dual perspective of convex optimization. We introduce the novel coupled continuous max-flow model and demonstrate that it is dual or equivalent to the studied convex minimization problem (5). With help of the introduced coupled continuous max-flow model, we prove the computed global optimum of the convex relaxation problem (5) also solve its original combinatorial optimization problem (4) globally and exactly. In addition, the coupled continuous max-flow model derives a new and efficient algorithm to (5) without directly tackling its challenging nonsmooth function terms, i.e. the total variation functions in (1) and the $L_{1}$-norm functions in (2) and (3). 


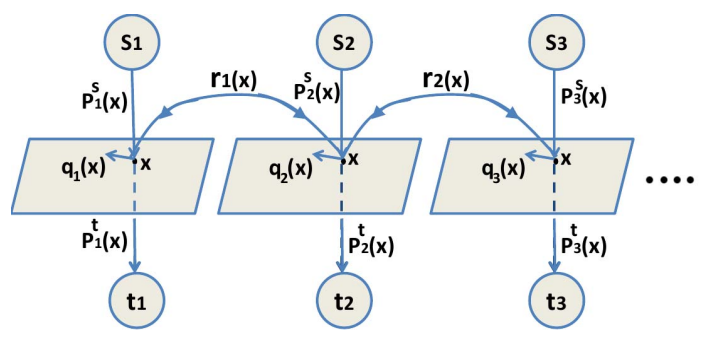

(a)

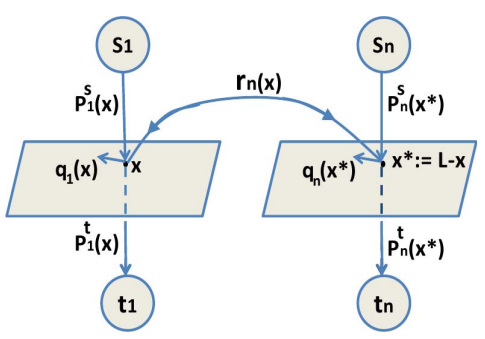

(b)

Figure 2. Flow-maximization configurations: (a) shows the flow-maximization configuration between two adjacent slices; (b) shows the flow-maximization configuration between the last and first slices.

Coupled Continuous Max-Flow Model We first introduce the new spatially continuous configuration of flows (as illustrated in Fig. 2 (a) and (b)), such that

- For each image slice $\mathcal{S}_{i}, i=1 \ldots n$, two additional flow terminals: the source $s_{i}$ and the sink $t_{i}$, are added; we link the source $s_{i}$ to each pixel $x$ in $\mathcal{S}_{i}$ and there is a flow $p_{i}^{s}(x)$ streaming from $s_{i}$ to $x$; we also link each pixel $x \in \mathcal{S}_{k}$ to the sink $t_{i}$ and there is a flow $p_{k}^{t}(x)$ streaming from $x$ to $t_{i}$; within $\mathcal{S}_{i}$, there is a local vector flow field $q_{i}(x) \in \mathbb{R}^{2}$ around $x$.

- Between two adjacent slices $\mathcal{S}_{i}$ and $\mathcal{S}_{i+1}, i=1 \ldots n-$ 1 , we link $x \in \mathcal{S}_{i}$ to the same pixel $x \in \mathcal{S}_{i+1}$ and there is a flow $r_{i}(x)$ streaming in both directions. Between the last slice $\mathcal{S}_{n}$ and the first slice $\mathcal{S}_{1}$, we link the pixel $x:=\left(x_{1}, x_{2}\right) \in \mathcal{S}_{1}$ to the pixel $\left(L-x_{1}, x_{2}\right) \in \mathcal{S}_{n}$ and there is a flow $r_{n}(x)$ streaming in both directions.

With the above flow settings (shown in Fig. 2 (a) and (b)), we introduce the new coupled continuous max-flow model, which maximizes the total amount of flows streaming from the $n$ sources $s_{1} \ldots s_{n}$, such that

$$
\max _{p^{s}, p^{t}, q, r} \sum_{i=1}^{n} \int_{\Omega} p_{i}^{s}(x) d x
$$

subject to the following flow constraints

- Capacity constraints on source and sink flows:

$$
p_{i}^{s}(x) \leq C_{i}^{s}(x), p_{i}^{t}(x) \leq C_{i}^{t}(x) ; i=1 \ldots n ;
$$

- Capacity constraints on spatial flows:

$$
\left|q_{i}(x)\right| \leq g_{i}(x), i=1 \ldots n ;
$$

- Capacity constraints on coupled flows:

$$
\left|r_{i}(x)\right| \leq \alpha, i=1 \ldots n ;
$$

- Flow conservation constraints: all the flows at each pixel of every slice are balanced, i.e. for each of the last $n-1$ slices $\mathcal{S}_{i}, i=2 \ldots n$, at each position $x \in \mathcal{S}_{i}$, it must suffice

$$
\rho_{i}(x):=\left(\operatorname{div} q_{i}-p_{i}^{s}+p_{i}^{t}+r_{i}-r_{i-1}\right)(x)=0 ;
$$

and for the first slice $\mathcal{S}_{1}$, at each position $x:=$ $\left(x_{1}, x_{2}\right) \in \mathcal{S}_{1}$, the total flow balance is evaluated by

$$
\rho_{1}(x):=\left(\operatorname{div} q_{1}-p_{1}^{s}+p_{1}^{t}+r_{1}\right)(x)-r_{n}\left(L-x_{1}, x_{2}\right)
$$

and it must suffice

$$
\rho_{1}(x)=0 .
$$

Primal and Dual Formulations Introduce the multiplier functions $u_{i}(x), i=1 \ldots n$, to the linear equalities (11) and (10) w.r.t. the flow conservation conditions, we then have the equivalent primal-dual model of (6) such that

$$
\min _{u_{1} \ldots u_{n}} \max _{p^{s}, p^{t}, q, r} \sum_{i=1}^{n} \int_{\Omega} p_{i}^{s}(x) d x+\sum_{i=1}^{n}\left\langle u_{i}, \rho_{i}\right\rangle
$$

subject to the flow capacity constraints (7) - (9).

By variational analysis, we can prove the following dualities or equivalences:

Proposition 1. The proposed coupled continuous max-flow model (6) is dual or equivalent to the convex relaxation problem (5), and also the primal-dual model (12), i.e.

$$
\text { (6) } \Longleftrightarrow(5) \Longleftrightarrow(12) \text {. }
$$

\subsection{Global and Exact Optimization of (4)}

With help of the coupled continuous max-flow model (6), we can prove

Proposition 2. Let $\left(u_{1}^{*}(x), \ldots u_{n}^{*}(x)\right) \in[0,1]$ be the global optimum of the convex relaxation problem (5), the thresholds $u_{i}^{\gamma}(x) \in\{0,1\}, i=1 \ldots n$, by any $\gamma \in[0,1)$, where

$$
u_{i}^{\gamma}(x)=\left\{\begin{array}{ll}
1, & \text { when } u_{k}^{*}(x)>\gamma \\
0, & \text { when } u_{k}^{*}(x) \leq \gamma
\end{array}, i=1 \ldots n,\right.
$$

solve the original binary-constrained co-segmentation problem (4) globally and exactly.

The main proof steps of Prop. 1 and Prop. 2 are referred to $[20,21,2]$ and omitted here due to the limit space. 


\section{Coupled Continuous Max-Flow Algorithm}

Through Prop. 2, we see that the global optimum of the challenging combinatorial optimization problem (4) can be achieved by thresholding the optimum of its convex relaxation (5) with any parameter $\gamma \in[0,1)$. On the other hand, in view of Prop. 1, it is also easy to see that the optimum of such convex relaxation problem (5) is just given by the optimal multipliers to the corresponding flow conservation conditions (10)-(11). Indeed, this allows to directly derive the coupled continuous max-flow algorithm based on the coupled continuous max-flow formulation (6) and the modern augmented Lagrangian algorithm [4], which computes both the maximum total flows from the $n$ sources and the optimum continuous labeling functions $u_{1 \ldots n}(x) \in[0,1]$. Especially, we will see the new coupled continuous max-flow algorithm is efficient and successfully avoids directly tackling the non-smooth function terms of (5).

In view of (12), we define the augmented Lagrangian function as follows:

$L_{c}\left(u, p^{s, t}, q, r\right):=\sum_{i=1}^{n}\left\{\int_{\Omega} p_{i}^{s}(x) d x+\left\langle u_{i}, \rho_{i}\right\rangle-\frac{c}{2}\left\|\rho_{i}\right\|^{2}\right\}$

where $c>0$ is constant. By means of the augmented Lagrangian algorithm, we propose the coupled continuous max-flow algorithm such that, at each $k$-th iteration,

1. Maximize $L_{c}\left(u, p^{s, t}, q, r\right)$ over the spatial flows $\left|q_{i}(x)\right| \leq g(x), i=1 \ldots n$, while fixing the other variables $\left(u, p^{s, t}, r\right)^{k}$, which amounts to

$$
q_{i}^{k+1}:=\underset{\left|q_{i}(x)\right| \leq g(x)}{\arg \max }-\frac{c}{2}\left\|\operatorname{div} q_{i}-F_{i}^{k}\right\|^{2},
$$

where $F_{i}^{k}(x)$ is fixed. This can be computed by the gradient-projection iteration:

$$
q_{i}^{k+1}=\operatorname{Proj}_{\left|q_{i}(x)\right| \leq g(x)}\left(q_{i}^{k}+\tau \nabla\left(\operatorname{div} q_{i}^{k}-\left(F_{i}^{k}\right)\right) ;\right.
$$

where $\tau>0$ is some step-size for convergence [6].

2. Maximize $L_{c}\left(u, p^{s, t}, q, r\right)$ over the source flows $p_{i}^{s}(x) \leq C_{i}^{s}(x), i=1 \ldots n$, while fixing the other variables $\left(u, p^{t}, q, r\right)^{k}$, which amounts to

$$
\left(p_{i}^{s}\right)^{k+1}:=\underset{p_{i}^{s}(x) \leq C_{i}^{s}(x)}{\arg \max } \int_{\Omega} p_{i}^{s} d x-\frac{c}{2}\left\|p_{i}^{s}-G_{i}^{k}\right\|^{2},
$$

where $G_{i}^{k}(x)$ is fixed. This can be solved exactly by:

$$
\left(p_{i}^{s}\right)^{k+1}(x)=\min \left(G_{i}^{k}(x)+1 / c, C_{i}^{s}(x)\right) .
$$

3. Maximize $L_{c}\left(u, p^{s, t}, q, r\right)$ over $p_{i}^{t}(x) \leq C_{i}^{t}(x), i=$ $1 \ldots n$, while fixing the other variables $\left(u, p^{s}, q, r\right)^{k}$, which amounts to

$$
\left(p_{i}^{t}\right)^{k+1}:=\underset{p_{i}^{t}(x) \leq C_{i}^{t}(x)}{\arg \max }-\frac{c}{2}\left\|p_{i}^{t}-H_{i}^{k}\right\|^{2},
$$

where $H_{1}^{k}(x)$ is fixed. This can be solved exactly by:

$$
\left(p_{i}^{t}\right)^{k+1}(x)=\min \left(H_{i}^{k}(x), C_{i}^{t}(x)\right) .
$$

4. Maximize $L_{c}\left(u_{1,2}, p_{s}^{1,2}, p_{t}^{1,2}, q_{1,2}, r\right)$ over the coupled flow field $|r(x)| \leq \beta$ by fixing $\left(q_{1,2}, p_{s}^{1,2}, p_{t}^{1,2}\right)^{k+1}$ and $\left(u_{1,2}\right)^{k}$, which gives

$$
r^{k+1}:=\underset{|r(x)| \leq \beta}{\arg \max }-\frac{c}{2}\left\|r+J_{1}^{k}\right\|^{2}-\frac{c}{2}\left\|r-J_{2}^{k}\right\|^{2},
$$

where $J_{1}^{k}=\operatorname{div} q_{1}^{k+1}-\left(p_{s}^{1}\right)^{k+1}+\left(p_{t}^{1}\right)^{k+1}-\left(u_{1}\right)^{k} / c$ and $J_{2}^{k}=\operatorname{div} q_{2}^{k+1}-\left(p_{s}^{2}\right)^{k+1}+\left(p_{t}^{2}\right)^{k+1}-\left(u_{2}\right)^{k} / c$. It can be computed exactly by

$$
r^{k+1}=\left(J_{2}-J_{1}\right) / 2 ;
$$

5. Update the labeling functions $u_{i}^{k+1}(x), i=1 \ldots n$, by

$$
u_{1}^{k+1}(x)=u_{i}^{k}(x)-c \rho_{i}^{k+1}(x) .
$$

Let $k=k+1$ and repeat the above steps until convergence is achieved.

In practice, the experiments show only one gradientprojection iteration (15) is needed to achieve convergence, which greatly improves numerical efficiency.

\section{Experiments and Results}

Settings: Twenty 3D endfiring TRUS prostate images were used to validate the proposed method. These images were acquired with a rotational scanning 3D TRUSguided prostate biopsy system, which made use of a commercially available end-firing TRUS transducer (Philips, Bothell WA). Each 3D image had $448 \times 448 \times 350$ voxels of size $0.19 \times 0.19 \times 0.19 \mathrm{~mm}^{3}$, and was resliced rotationally to $302 \mathrm{D}$ slices with a reslicing step angle of $6^{\circ}$. A mean shape (green contour in Fig.1 (b)) was used to initialize the segmentation of the first slice by visually coinciding its center (red dot in Fig.1 (b)) with the prostate centroid. The mean shape was learned from twenty manually segmented 2D prostate transverse images, which were not used in the validation procedure.

Our segmentation method was evaluated using the following metrics by comparing our computation results to manual segmentations: Dice similarity coefficient (DSC), the mean absolute surface distance (MAD) and maximum absolute surface distance (MAXD) [9]. The proposed method, denoted by $M_{G O}$, was also compared to other rotational-resliced segmentation methods: active contour based method $\left(M_{A C}\right)$ [8] and level set based method $\left(M_{L S}\right)$ [14]. The proposed coupled max-flow algorithm was implemented using GPU (CUDA, NVIDIA) and the user interface in Matlab. The experiments were conducted on 
a Windows desktop with Intel i7-2600 CPU and NVIDIA GTX 580 GPU.

Shape Priors: In this study, a 2D statistical shape model, learned by principal component analysis (PCA) $[7,16,18,19]$ over a dataset with $N_{d}$ samples, was introduced to facilitate segmenting the first slice, hence all the slices in turn. The signed distance function (SDF) $\psi(x)$ was chosen as the representation of the sampled prostate contour to build such a shape prior, where the mean and variance of all contour samples were approximated by PCA:

The mean SDF $\bar{\psi}$ was subtracted from each $\psi$ and placed as a column vector in a $N_{d} \times m$-dimensional matrix $Q$ where $m$ is the number of spatial dimensions. Using singular value decomposition (SVD), we have $Q=U \Sigma V^{T}$ such that $U$ is a matrix whose column vectors represent the set of orthogonal modes of shape variation, and $\Sigma$ is a diagonal matrix of corresponding singular values. An object shape can be approximated by the $k$ principal components and a $k$-dimensional coefficient vector (where $k<m$ ) $\alpha_{i}$ : $\tilde{\psi}=U_{k} \alpha_{i}+\bar{\psi}$, where $U_{k}$ is a $N_{d} \times k$ matrix consisting of the first $k$ columns of matrix $U$. Under the assumption of a Gaussian distribution of object represented by $\alpha_{i}$, the probability of a certain shape is computed as:

$$
p\left(\alpha_{i}\right)=\frac{1}{\sqrt{(2 \pi)^{k}\left|\Sigma_{k}\right|}} \exp \left[-\frac{1}{2} \alpha_{i}^{T} \Sigma_{k}^{1} \alpha_{i}\right]
$$

We define all the cost functions $C_{i}^{s}(x):=-\log \phi_{f g}(x)$ and $C_{i}^{t}(x)=-\log \phi_{b g}(x), i=1 \ldots n$, where $\phi_{f g}(x)$ and $\phi_{b g}(x)$ are the intensity PDFs w.r.t. foreground and background, estimated by sampled pixels in one slice or learned through the training data. For the first slice $\mathcal{S}_{1}$, its two cost functions are updated as:

$$
\left\{\begin{array}{c}
\tilde{C}_{1}^{s}(x)=C_{1}^{s}(x)+w_{1} \tilde{u}_{1}(x) \alpha_{i}^{T} \Sigma_{k}^{1} \alpha_{i} \\
\tilde{C}_{1}^{t}(x)=C_{1}^{t}(x)+w_{1}\left(1-\tilde{u}_{1}(x)\right) \alpha_{i}^{T} \Sigma_{k}^{1} \alpha_{i}
\end{array}\right.
$$

where $w_{1}>0$ is constant and $\tilde{u}_{1}(x)$ is the labeling function of the segmentation of $\mathcal{S}_{1}$ from the previous iteration.

Accuracy: Careful initializations were required by the $M_{A C}$ and $M_{L S}$ methods, where four to eight points on the prostate boundary in the first slice were selected to construct the initial contour by the cardinal splines [8]. In contrast, only an approximate center point was selected to initialize the proposed $M_{G O}$ method. The validation shown in Table. 1 clearly indicates that, compared to the $M_{L S}$ method and the $M_{A C}$ method, the proposed $M_{G O}$ method achieved the best accuracy result in terms of DSC: $93.7 \pm 2.1 \%$, MAD: $1.12 \pm 0.4 m m(5.9 \pm 2.1$ voxels $)$ and MAXD: $3.15 \pm 0.65 \mathrm{~mm}(16.6 \pm 3.4$ voxels $)$, as well as the least user interactions (Fig. 3 illustrates an example computed by the proposed $M_{G O}$ method). Also, Table. 1 shows that the active contour based method $\left(M_{A C}\right)$ performed the poorest with our dataset; the $M_{L S}$ method can also obtain

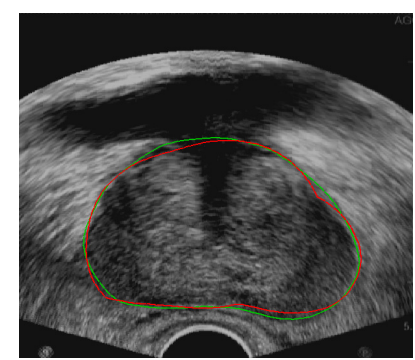

(a)

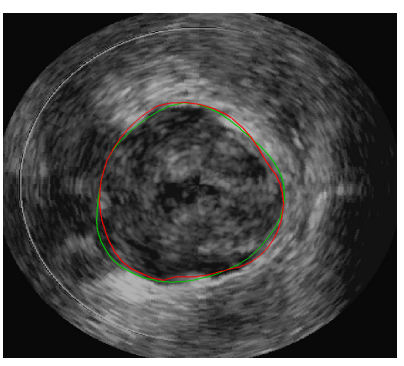

(c)

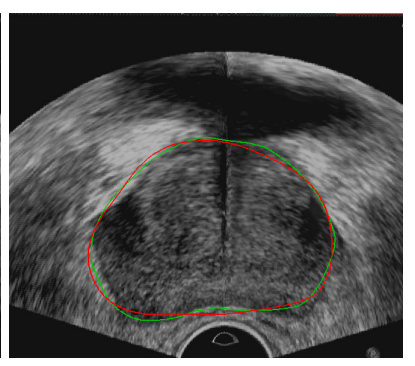

(b)

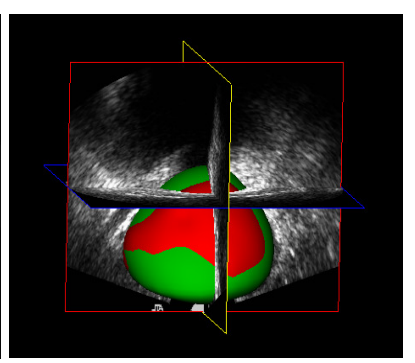

(d)
Figure 3. 3D prostate segmentation (green contour) by the proposed $M_{G O}$ method and manual segmentation (red contour): (a) transverse view; (b) sagittal view; (c) coronal view; (d) orthogonal view overlapped with the manual segmentation surface.

a comparable segmentation accuracy to the $M_{G O}$ method, due to the contributions of several additional energy terms such as anchor points, shape constraint and strong statistical information about the local region; however, the $M_{L S}$ method took much longer computational time and interactions than the $M_{G O}$ method.

Reliability: It should be noted that the segmentation accuracy of the methods $M_{A C}$ and $M_{L S}$ relies on the segmentation accuracy of the first slice. Segmentation errors appearing in one slice will be introduced to the segmentation of the next slice and so on, thereby larger errors can be accumulated to affect the segmentation of the 3D TRUS image. In the experiments of $M_{A C}$ and $M_{L S}$, the first slice and its initial contour should be carefully determined by the user in order to decrease the associated segmentation errors as much as possible. However, due to poor image quality, it is still challenging to locate the correct prostate boundary in some cases based only on the information of a single slice, even incorporating some high-level interaction (initial boundary points). For examples, when it appears that the overlapped area between the prostate and urethral entrance (the arrow shown in Fig. 4 (a)) or seminal vesicle (the arrow shown in Fig. 4 (b)), it is hard to distinguish the correct prostate boundary even for radiologists without the image information about its neighboring slices. Fig. 4 shows the segmentation results of $M_{A C}$ and $M_{L S}$ with poor initialization: their segmentations of the first slice with the same initialization are illustrated in Fig.4(a) and (b), re- 


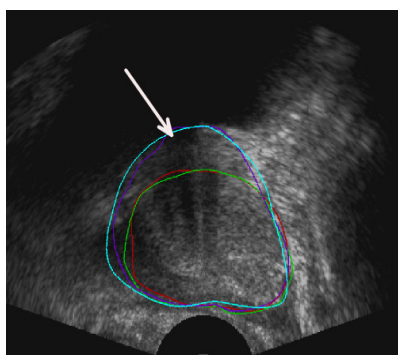

(a)

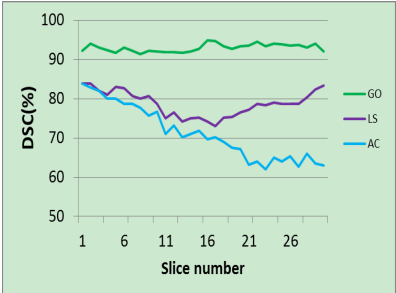

(c)

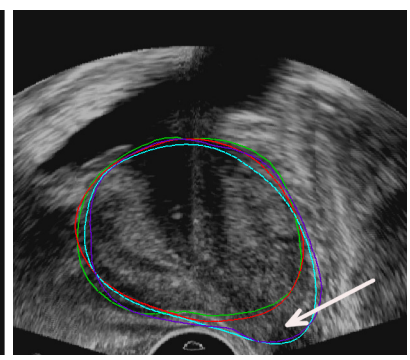

(b)

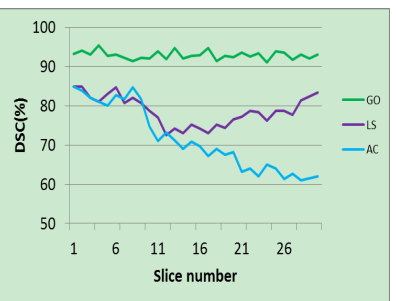

(d)
Figure 4. Segmentation results with poor initialization. Green: $M_{G O}$; red: manual segmentation; blue: $M_{L S}$; purple: $M_{A C}$. (a) segmentation for the first slice which contains the urethral region (arrow shows); (b) segmentation for the first slice which contains the seminal vesicle region (arrow shows); (c) DSC for all resliced slices based on the segmentation in (a); (d) DSC for all resliced slices based on the segmentation in (b);

spectively. With the result for the first slice, the segmentation accuracy for all slices, computed by $M_{A C}$, decreases greatly with the progress of the propagation (blue curve in Fig.4(c) and (d)). The $M_{L S}$ method can decrease the accumulated errors by propagating in two different directions, however, its DSC values demonstrate the same trend as the $M_{A C}$ method in each direction (purple curves in Fig.4 (c) and (d)). In contrast, the proposed $M_{G O}$ approach makes use of the global rotational correlation between all adjacent slices, which jointly adjusts the segmentation of each slice in a global way, hence performs much more reliably with the poor image quality and initialization (green curves in Fig. 4(c) and (d)) compared to the other two methods.

Reproducibility: To evaluate the variability of the proposed method, 10 3D images were randomly selected. Each image was segmented three times by the same observer for assessing intra-observer variability. A DSC of $93.0 \pm 2.5 \%$ and a coefficient-of-variation $(C V)$ [23] of $2.7 \%$ was obtained. ANOVA analysis with a single factor showed that there is no statistically significant difference between these three segmentations ( $p=0.95, F=0.32$ ). These ten images were also segmented three times by three untrained observers who were blinded to patient identity for assessing inter-observer variability. The proposed method yielded a DSC of $93.5 \pm 2.1 \%, 92.6 \pm 3.1 \%$ and $92.3 \pm 3.2 \%$, and a $C O V$ of $2.3 \%, 3.3 \%$ and $3.5 \%$, respectively. ANOVA analysis with a single factor failed to demonstrate a statis-

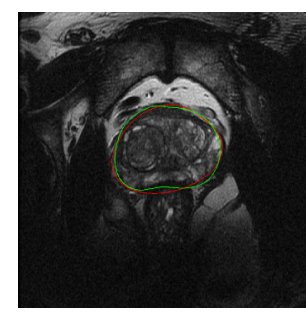

(a)

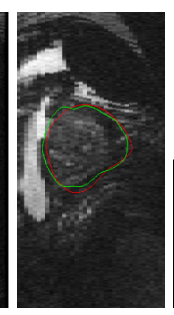

(b)

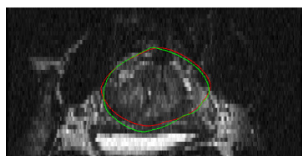

(c)

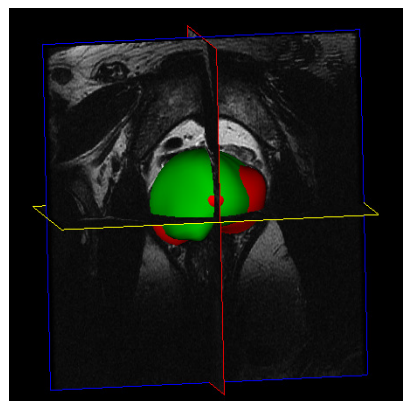

(d)

Figure 5. A prostate segmentation result from a 3D MR image. Green boundary: algorithmic result, red boundary: manual segmentation result. (a) transverse view; (b) sagittal view; (c) coronal view; (d) orthogonal view overlapped with the segmented surface.

tically significant difference between these three segmentations ( $p=0.51, F=0.82)$.

Efficiency: The mean computation time of the proposed $M_{G O}$ method was determined by 5 repeated experiments for each 3D TRUS image. As shown in Table.1, the shortest computation time was required by the proposed method: the mean segmentation time was $0.45 \pm 0.1 \mathrm{~s}$, significantly less than $55 \pm 3.5 \mathrm{~s}$ for the $M_{L S}$ method and $22.5 \pm 2.6 \mathrm{~s}$ for the $M_{A C}$ method. The total processing time was about $2.45 \mathrm{~s}$ including about $2 \pm 1 s$ for initialization.

Application in 3D Prostate MRIs: We also applied the proposed method to segment 2 3D T2-weighted MR prostate images using a body coil, which were acquired at a size of $291 \times 341 \times 38$ voxels with a voxel size of $0.27 \times 0.27 \times 2.2 \mathrm{~mm}^{3}$. For simplicity, the rotational axis and first slice were manually determined in the transverse view. Our method obtained the DSC accuracy of $89.2 \%$ and $90.5 \%$, respectively. Fig. 5 shows that the segmentation boundary agrees well with the manual segmentation, even in the sagittal view (Fig. 5 (b)), and coronal view (Fig. 5 (c)) where the resolution is lower than the transverse view.

\section{Conclusions}

In this paper, we propose a novel global optimized resliced approach to the computationally challenging $3 \mathrm{D}$ endfiring TRUS prostate images by enforcing the inherent rotational symmetry of prostate shapes, which jointly segments a series of 2D reslices in a global sense. Moreover, 
Table 1. Overall performance results for twenty patient images

\begin{tabular}{cccccc}
\hline & DSC (\%) & MAD (mm (vx)) & MAXD (mm (vx)) & Time (s) & Initial points \\
\hline \multirow{2}{*}{$M_{A C}$} & \multirow{2}{*}{$86.5 \pm 4.5$} & $2.28 \pm 0.82$ & $6.23 \pm 2.68$ & \multirow{2}{*}{$22.5 \pm 2.6$} & $4-8$ \\
& & $(12.0 \pm 4.3)$ & $(32.8 \pm 14.1)$ & & \\
$M_{L S}$ & \multirow{2}{*}{$93.0 \pm 3.6$} & $1.18 \pm 0.36$ & $3.44 \pm 0.80$ & $55 \pm 3.5$ & $4-8$ \\
& & $(6.2 \pm 1.9)$ & $(18.1 \pm 4.2)$ & & \\
\multirow{2}{*}{$\mathbf{M}_{\text {GO }}$} & \multirow{2}{*}{$\mathbf{9 3 . 7} \pm \mathbf{2 . 1}$} & $\mathbf{1 . 1 2} \pm \mathbf{0 . 4}$ & $\mathbf{3 . 1 5} \pm \mathbf{0 . 6 5}$ & $\mathbf{0 . 4 5} \pm \mathbf{0 . 1}$ & $\mathbf{1}$ \\
& & $\mathbf{( 5 . 9} \pm \mathbf{2 . 1})$ & $\mathbf{( 1 6 . 6 \pm \mathbf { 3 . 4 } )}$ & & \\
\hline
\end{tabular}

we proposed a novel coupled continuous max-flow model, which not only provides a powerful mathematical tool to analyze the proposed optimization problem but also directly derives a new and efficient duality-based algorithm in numerical practices.

Acknowledgments The authors are grateful for funding from the Canadian Institutes of Health Research (CIHR) and the Ontario Institute of Cancer Research (OICR). E. Ukwatta acknowledges the support from Natural Sciences and Engineering Research Council (NSERC) Canada Research Scholarship (CGS). A. Fenster holds a Canada Research Chair in Biomedical Engineering, and acknowledges the support of the Canada Research Chair Program.

\section{References}

[1] B. Appleton and H. Talbot. Globally minimal surfaces by continuous maximal flows. IEEE Trans. Pattern Anal. Mach. Intell., 28(1):106-118, 2006.

[2] E. Bae, J. Yuan, X.-C. Tai, and Y. Boycov. A study on continuous max-flow and min-cut approaches part ii: Multiple linearly ordered labels. Technical report CAM-10-62, UCLA, 2010.

[3] J. Bax, D. Cool, L. Gardi, K. Knight, D. Smith, J. Montreuil, S. Sherebrin, C. Romagnoli, and A. Fenster. Mechanically assisted 3D ultrasound guided prostate biopsy system. Med. Phys., 35(12):5397-5410, 2008.

[4] D. P. Bertsekas. Nonlinear Programming. Athena Scientific, September 1999.

[5] D. Beyersdorff, A. Winkel, B. Hamm, S. Lenk, S. A. Loening, and M. Taupitz. MR imaging-guided prostate biopsy with a closed MR unit at $1.5 \mathrm{~T}$ : initial results. Radiology, 234(2):576-581, Feb 2005.

[6] A. Chambolle. An algorithm for total variation minimization and applications. Journal of Mathematical Imaging and Vision, 20(1):89-97, January 2004.

[7] D. Cremers. Dynamical statistical shape priors for level setbased tracking. IEEE Trans. Pattern Anal. Mach. Intell., 28(8):1262-1273, 2006.

[8] M. Ding, B. Chiu, I. Gyacskov, X. Yuan, M. Drangova, D. B. Downey, and A. Fenster. Fast prostate segmentation in 3D TRUS images based on continuity constraint using an autoregressive model. Med. Phys., 34(11):4109-4125, 2007.

[9] C. Garnier, J.-J. Bellanger, K. Wu, H. Shu, N. Costet, R. Mathieu, R. de Crevoisier, and J.-L. Coatrieux. Prostate segmentation in HIFU therapy. IEEE Trans. Med. Imag., 30(3):792-803, 2011.

[10] N. Hu, D. B. Downey, A. Fenster, and H. M. Ladak. Prostate boundary segmentation from 3D ultrasound images. Med. Phys., 30(7):1648-1659, Jul 2003.

[11] A. Jemal, R. Siegel, J. Xu, and E. Ward. Cancer statistics, 2010. CA Cancer J Clin, 60(5):277-300, 2010.

[12] A. Krieger, R. Susil, C. Menard, J. Coleman, G. Fichtinger, E. Atalar, and L. Whitcomb. Design of a novel MRI compatible manipulator for image guided prostate interventions. IEEE Trans. BME, 52(2):306 -313, 2005.

[13] S. S. Mahdavi, M. Moradi, X. Wen, W. J. Morris, and S. E. Salcudean. Evaluation of visualization of the prostate gland in vibro-elastography images. Medical Image Analysis, 15(4):589-600, 2011.

[14] W. Qiu, J. Yuan, E. Ukwatta, and D. T. A. Fenster. Rotational-slice-based prostate segmentation using level set with shape constraint for $3 \mathrm{~d}$ end-firing trus guided biopsy. In MICCAI,, Part 1, LNSC 7510, 2012, pages 537-544, 2012.

[15] M. Rifkin. Ultrasound of the prostate: imaging in the diagnosis and therapy of prostatic disease. Lippincott-Raven Publishers, 1997.

[16] A. Tsai, J. Yezzi, A., W. Wells, C. Tempany, D. Tucker, A. Fan, W. Grimson, and A. Willsky. A shape-based approach to the segmentation of medical imagery using level sets. IEEE Trans. Med. Imaging, 22(2):137 -154, 2003.

[17] I. B. Tutar, S. D. Pathak, L. Gong, P. S. Cho, K. Wallner, and Y. Kim. Semiautomatic 3D prostate segmentation from TRUS images using spherical harmonics. IEEE Trans. Med. Imaging, 25(12):1645-1654, 2006.

[18] J. Yang and J. S. Duncan. 3d image segmentation of deformable objects with joint shape-intensity prior models using level sets. Medical Image Analysis, 8(3):285-294, 2004.

[19] J. Yang, L. H. Staib, and J. S. Duncan. Neighbor-constrained segmentation with level set based 3-d deformable models. IEEE Trans. Med. Imaging, 23(8):940-948, 2004.

[20] J. Yuan, E. Bae, and X. Tai. A study on continuous max-flow and min-cut approaches. In CVPR 2010.

[21] J. Yuan, E. Bae, X.-C. Tai, and Y. Boykov. A continuous max-flow approach to potts model. In ECCV, 2010.

[22] Y. Zhan, D. Shen, J. Zeng, L. Sun, G. Fichtinger, J. Moul, and C. Davatzikos. Targeted prostate biopsy using statistical image analysis. IEEE Trans. Med Imag, 26(6):779-788.

[23] K. H. Zou and M. P. Mcdermott. Higher-moment approaches to approximate interval estimation for a certain intraclass correlation coefficient. Statistics in Medicine, 18(15):20512061, 1999. 